\title{
Evaluation of the Efficacy of Three Different Treatment Modalities in the Management of Dentinal Hypersensitivity: A Comparative Study
}

\author{
Mohammad Jalaluddin ${ }^{1}$, Sultan A Almalki ${ }^{2}$
}

\begin{abstract}
Aim:The aim of the present study was to assess the efficacy of three different treatment modalities in the management of dentinal hypersensitivity (DH).

Materials and methods: A split-mouth, randomized clinical study with a total of 30 (12 males and 18 females) patients aged between 18 years and 45 years, with 60 hypersensitive teeth, was conducted. The extent of DH was assessed using the tactile stimuli test and cold air blast. A total of 60 hypersensitive teeth from 30 patients were randomly divided into three groups: group I, hypersensitive teeth treated with iontophoresis with acidulated phosphate fluoride (APF) gel; group II, hypersensitive teeth treated with a diode laser (DL); and group III, hypersensitive teeth treated with fluoride varnish. All the three groups were assessed for DH using visual analogue scale (VAS) at baseline and on days 15 and 30 after the initial treatment. An analysis of variance (ANOVA) repeated measure analysis was done and $p<0.05$ was considered as statistically significant. Results: Out of the 30 enrolled patients, $18(60 \%)$ were females and $12(40 \%)$ were males. Most of them (43.3\%) were aged between 36 and 45 years. A majority of the included teeth were anterior [32(53.3\%)]. The mean VAS scores among three groups for tactile stimulus response showed a maximum reduction $(1.90 \pm 1.20)$ of $\mathrm{DH}$ in group II with a DL after 30 days. Similar reductions were recorded in the mean VAS scores among three groups for cold air blast stimulus response, with maximum reduction $(2.02 \pm 0.10)$ of $\mathrm{DH}$ in group II after 30 days. A statistically significant difference was observed among all the three groups.

Conclusion: The present study concluded that a $980 \mathrm{~nm}$ wavelength DL demonstrated significantly greater reduction of DH followed by iontophoresis with APF and fluoride varnish, respectively.

Keywords: Dentin hypersensitivity, Diode laser, Fluoride varnish, lontophoresis.

World Journal of Dentistry (2019): 10.5005/jp-journals-10015-1626
\end{abstract}

\section{INTRODUCTION}

Dentinal hypersensitivity $(\mathrm{DH})$ is fairly a common issue encountered in periodontal clinics. DH is defined by a sharp pain that lasts for a short duration. This $\mathrm{DH}$-associated pain arises from bare dentin when stimulated by heat, cold, and touch, as well as osmotic, evaporative, or chemical reactions, and cannot be characterized as any other dental condition. DH may naturally develop in teeth with gingival recession, where the root becomes unmasked to the oral environment, or it may eventually arise after scaling and root planning procedures and periodontal surgeries. ${ }^{1}$

The diverse available evidence reports a 3-57\% prevalence of $\mathrm{DH}$, with an estimated average of $15 \%$ in the adult population. The incidence of $\mathrm{DH}$ increases with age; however, most of the patients affected by $\mathrm{DH}$ are aged between second and fifth decades of life, with peak occurrences ranging from 30 to 40 years of age. Female predilection is more common. Sufferers of periodontal diseases are inclined to be affected more, with the frequency ranging from 72 to $98 \% .^{2,3}$

The causes of dentin exposure are manifold, such as attrition due to parafunctional habits; abrasion due to faulty tooth brushing; erosion by acids; and abfractions and gingival recession due to periodontal diseases, ultimately resulting in hypersensitivity. ${ }^{4}$

The pain associated with $\mathrm{DH}$ has been well explained by the most accepted hydrodynamic theory (Brannstrom and Astron, 1964). According to this theory, environmental trauma stimulates the movement of fluid within the dentinal tubules, which in turn disturbs the nerve terminals of the pulp, thus causing pain. ${ }^{5}$
${ }^{1}$ Department of Periodontics and Oral Implantology, Kalinga Institute of Dental Sciences, Kalinga Institute of Industrial Technology (Deemed to be University), Bhubaneswar, Odisha, India

${ }^{2}$ Department of Preventive Dental Sciences, College of Dentistry, Prince Sattam Bin Abdulaziz University, Alkharj, Kingdom of Saudi Arabia

Corresponding Author: Mohammad Jalaluddin, Department of Periodontics and Oral Implantology, Kalinga Institute of Dental Sciences, Kalinga Institute of Industrial Technology (Deemed to be University), Bhubaneswar, Odisha, India, Phone: +91 9338131843, e-mail: drjalal1979@gmail.com

How to cite this article: Jalaluddin M, Almalki SA. Evaluation of the Efficacy of Three Different Treatment Modalities in the Management of Dentinal Hypersensitivity: A Comparative Study. World J Dent 2019;10(3):202-206.

Source of support: Nil

Conflict of interest: None

The various agents that are most commonly used in the treatment of DH include anti-inflammatory agents; tubule sealants (resins and adhesives); protein precipitants (formaldehyde, silver nitrate, and strontium chloride); tubule-occluding agents (potassium oxalate, potassium nitrate, calcium hydroxide, and sodium fluoride [NaF]); and miscellaneous (lasers). ${ }^{6}$ Until today, numerous trials have been conducted using several agents, such as mouthwashes, dentifrices, gels, etc., to terminate this undesirable symptom. ${ }^{7}$ However, none of these have been proven to be effective 
in long term. Thus, the present study was conducted to evaluate the efficacy of three different $\mathrm{DH}$ treatment modalities.

\section{Materials and Methods}

A randomized split-mouth clinical trial was conducted in the Department of Periodontics, Kalinga Institute of Dental Sciences, Bhubaneswar, India. A total of 30 (12 males and 18 females) patients aged between 18 years and 45 years with 60 hypersensitive teeth were included this study. Patients included were free from diseases, no known allergies to commercial dental products, had not used any desensitizing toothpaste since last 3 months, and had no cavities or teeth restorations.

The patients who had poor periodontal health; debilitating systemic condition; previously treated for $\mathrm{DH}$; cavities, restorations, orthodontic appliance, crowns and bridges in the area of sensitivity; and hypersensitivity to the agents used in the present study were excluded from the study.

\section{Assessment of DH}

\section{Tactile Stimuli Test}

An examiner held the tip of the \#16 explorer at right angles to the dentin surface of the tooth that requires treatment and moved it horizontally with a sweeping motion. The examiner applied $10 \mathrm{~g}$ of force and increased it gradually, where a pain response was provoked. The examiner retested the teeth by applying a similar force and only those teeth that showed a positive response both the times were used as test teeth, and the amount of force used was recorded as threshold. With a negative pain response, the amount of force applied was increased by $2 \mathrm{~g}$ and the same process was performed again. The procedure was repeated until the patient response was positive. All evaluations were made by the same clinician (Fig. 1).

\section{Cold Air Blast}

An air syringe of the dental unit was used as an evaporative stimulus. The air was directed at the exposed tooth area for $3 \mathrm{~s}$ from a distance of $1 \mathrm{~cm}$ and perpendicular to the buccal site of the assigned teeth (Fig. 2).

The visual analogue scale (VAS) score was recorded for each patient at baseline, day 15 , and day 30 . The VAS is a 10-point scale with the anchor words "no pain $(0 \mathrm{~cm})$ " and "intolerable pain $(10 \mathrm{~cm})$ "

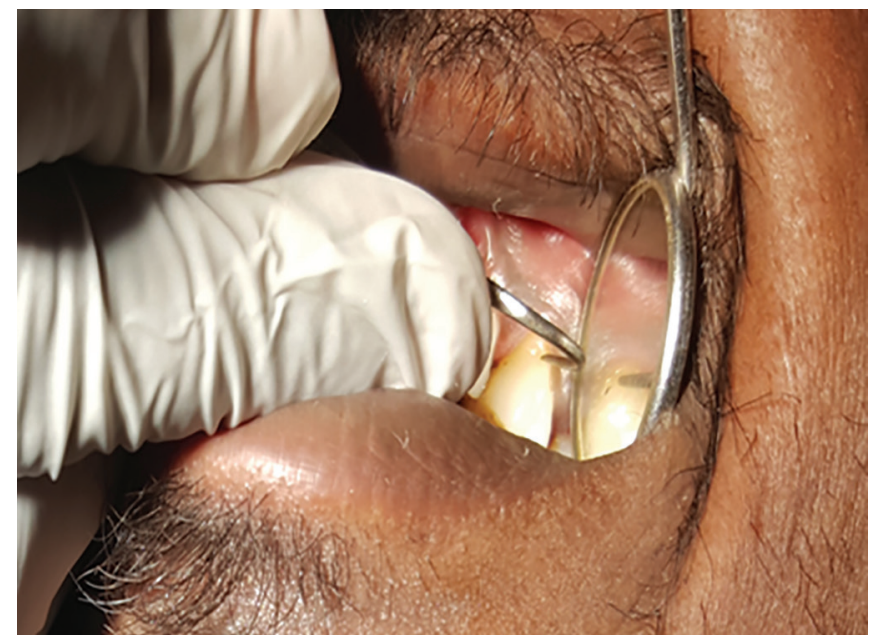

Fig. 1: Tactile stimuli test at the opposite ends. Each participant had to place a vertical mark on the VAS to indicate the intensity of his or her level of sensitivity after receiving stimuli.

A total of 60 teeth from 30 patients were randomly divided into three groups.

\section{Group I: Patients Treated with lontophoresis with APF Gel}

After isolating the tooth with cotton rolls and drying, a thin coat of APF gel was applied with a brush (one stroke of the brush). The iontophoresis unit (Desensitron $I^{\circledR}$ ) was turned on with the circuit being completed and a gradually increasing current was applied to the tooth until the patient sensed pain or sensitivity. Once this threshold was reached, the APF gel was reapplied and the procedure was followed again at a lower ampere current.

\section{Group II: Patients Treated with a Diode Laser (DL)}

The sensitive teeth were irradiated with a DL beam having a wavelength of $980 \mathrm{~nm}$, and a 320-micrometer core diameter optic fiber with $0.5 \mathrm{~W}$ output power. Each site was treated for $30 \mathrm{~s}$. The laser beam was directed at right angle to the tooth surface at three different points: mesiobuccal, buccal, and distobuccal.

\section{Group III: Patients Treated with Fluoride Varnish}

A thin coat of NaF varnish (Duraphat) was applied on the tooth surface with a disposable micro brush according to the manufacturer's instructions. After ensuring complete isolation, two to three coats of varnish were applied on the same day. The patients were asked to avoid carbonated drinks or food for $1 \mathrm{~h}$ after varnish application.

All the three groups were assessed for DH using the VAS at baseline, on day 15 , and on day 30 after the initial therapy.

\section{Statistical Analysis}

On the basis of various stimuli, the mean values of the clinical parameters were calculated for all the groups. The ANOVA repeated measure test was used to evaluate the scores obtained at baseline through the 30th day. The level of significance was considered to be $5 \%$ ( $p<0.05$, statistically significant).

\section{RESULTS}

Table 1 displays the distribution of age, gender, and tooth type among study participants. Of the 30 enrolled patients, 18 (60\%) were

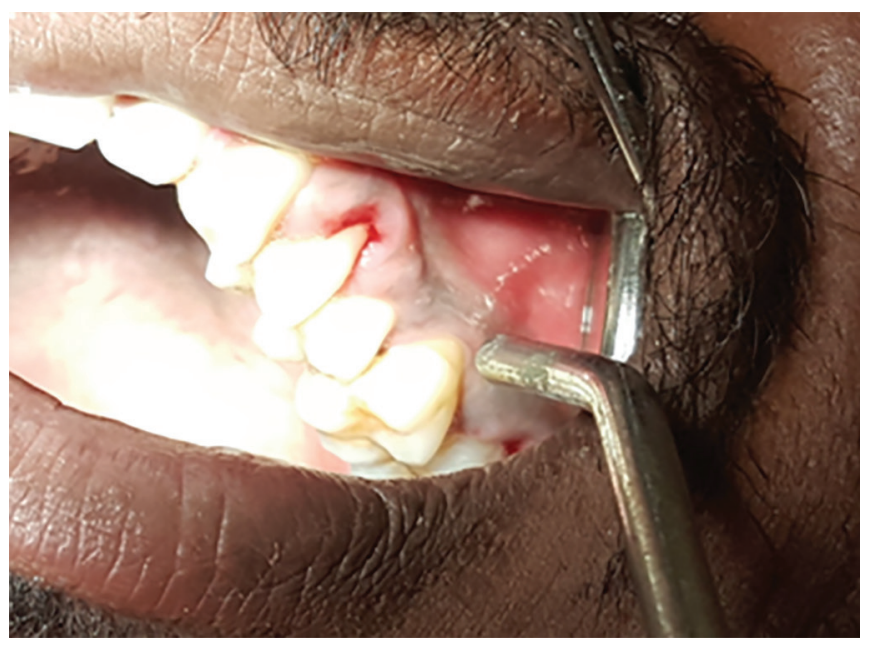

Fig. 2: Cold air blast test 
females and 12 (40\%) were males. About $23.4 \%$ of the participants were in the $18-25$ years age group, $33.3 \%$ were in the $26-35$ years age group, and $43.3 \%$ were in the $36-45$ years age group. Of the 60 included teeth, 53.3\% were anterior teeth, 20 (33.3\%) were premolars, and 8 (13.4\%) were molars.

Table 2 and Figure 3 show the mean VAS scores among the three groups for tactile stimulus response at various time intervals. The mean VAS score for group I (treated with iontophoresis with APF gel) was $6.28 \pm 1.03$ at baseline, which gradually reduced to $2.65 \pm 1.8$ with treatment after 30 days. A mean VAS score of $7.88 \pm 0.16$ was recorded at baseline for group II (treated with a $D L$ ), which reduced to $1.90 \pm 1.20$ with treatment after 30 days. A maximum reduction in the DH was seen in this group. Group III (treated with fluoride varnish) demonstrated a mean VAS score of $7.10 \pm 1.44$ at baseline

Table 1: Distribution of age, gender, and tooth type among study participants

\begin{tabular}{|c|c|c|}
\hline \multicolumn{2}{|c|}{ Variables } & \multirow{2}{*}{$\frac{n(\%)}{7(23.4)}$} \\
\hline Age & $18-25$ & \\
\hline & $26-35$ & $10(33.3)$ \\
\hline & $36-45$ & $13(43.3)$ \\
\hline \multirow[t]{2}{*}{ Gender } & Male & $12(40)$ \\
\hline & Female & $18(60)$ \\
\hline \multirow[t]{3}{*}{ Tooth type } & Anterior & $32(53.3)$ \\
\hline & Premolar & $20(33.3)$ \\
\hline & Molar & $8(13.4)$ \\
\hline
\end{tabular}

Table 2: Mean VAS scores among three groups for tactile stimulus response at different time intervals

\begin{tabular}{llllll}
\hline Groups & Time interval & $n$ & Mean $\pm S D$ & fvalue & $p$ value \\
\hline Group I: & Baseline & 20 & $6.28 \pm 1.03$ & 21.882 & 0.001 \\
lontophoresis & 15 days & 20 & $4.14 \pm 0.21$ & & \\
with APF gel & 30 days & 20 & $2.65 \pm 1.86$ & & \\
Group II: DL & Baseline & 20 & $7.88 \pm 0.16$ & 38.962 & 0.001 \\
& 15 days & 20 & $5.10 \pm 0.55$ & & \\
& 30 days & 20 & $1.90 \pm 1.20$ & & \\
Group III: Fluoride & Baseline & 20 & $7.10 \pm 1.44$ & 32.100 & 0.04 \\
varnish & 15 days & 20 & $6.02 \pm 0.21$ & & \\
& 30 days & 20 & $5.54 \pm 1.80$ & & \\
\hline
\end{tabular}

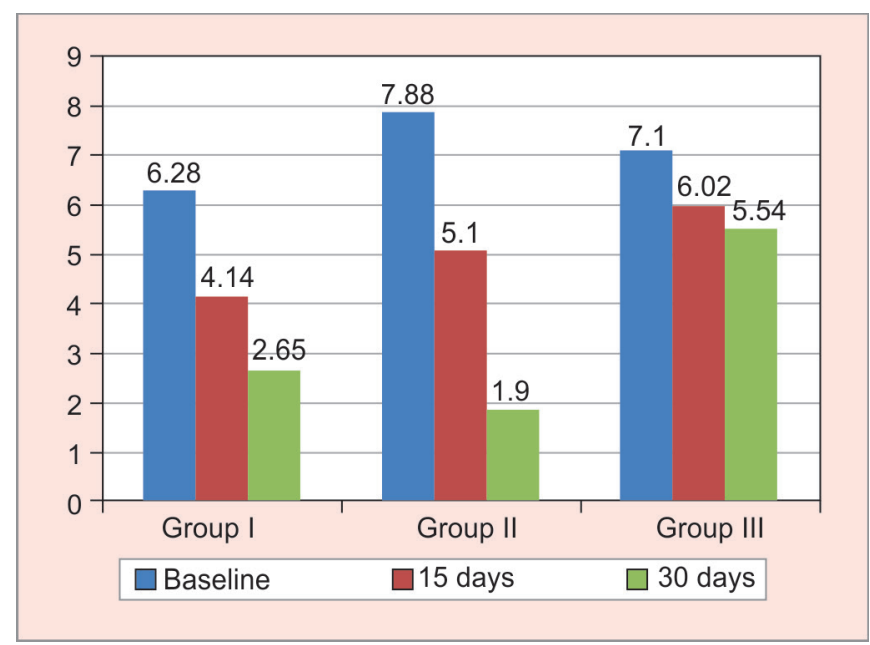

Fig. 3: Mean VAS scores among the three groups for tactile stimulus and a gradual reduction to $5.54 \pm 1.80$ after 30 days. A statistically significant difference was obtained among all the three groups.

The mean VAS scores among the three groups for cold air blast stimulus response at various time intervals are as follows: $7.11 \pm 0.78$ at baseline and $3.25 \pm 0.40$ after 30 days in group l; $6.17 \pm 0.98$ at baseline and $2.02 \pm 0.10$ after 30 days in group II; and $6.90 \pm 0.75$ at baseline and $4.06 \pm 0.30$ after 30 days in group III. A maximum reduction in the $\mathrm{DH}$ was seen in group II. Statistically significant difference was obtained in all the three groups (Table 3 and Fig. 4).

\section{Discussion}

It is possible to minimize $\mathrm{DH}$ by reducing the movement of dentinal fluid within the dentinal tubules and, thus, sequentially reducing the disturbance to the odontoblastic process. There are several ways by which dentinal tubules can be occluded: sealing the dentin surface, occluding their openings, or blocking the subsurface dentin. ${ }^{8}$

So far, there has been no gold standard for the treatment of $\mathrm{DH}$ and thus $\mathrm{DH}$ persists as a chronic dental problem. The most probable method of eliminating the pain associated with $\mathrm{DH}$ appears to be disruption of the transmission of stimuli to the nerve terminals that accompany the odontoblastic processes by minimizing the dentinal fluid movement within the tubules, which can be obtained either by constriction or by occlusion of tubule orifices. ${ }^{9}$ As $\mathrm{DH}$ of the cervical region is caused by several factors, multiple treatment modalities should be adapted to normalize the dentin sensitivity. ${ }^{10}$

Table 3: Mean VAS scores among three groups for cold air blast stimulus response at different time intervals

\begin{tabular}{llllll}
\hline Groups & Time interval & $n$ & Mean $\pm S D$ & fvalue & $p$ value \\
\hline Group I: & Baseline & 20 & $7.11 \pm 0.78$ & 39.142 & 0.001 \\
lontophoresis & 15 days & 20 & $5.01 \pm 0.22$ & & \\
with APF gel & 30 days & 20 & $3.25 \pm 0.40$ & & \\
Group II: DL & Baseline & 20 & $6.17 \pm 0.98$ & 41.051 & 0.001 \\
& 15 days & 20 & $4.49 \pm 0.39$ & & \\
& 30 days & 20 & $2.02 \pm 0.10$ & & \\
Group III: Fluoride & Baseline & 20 & $6.90 \pm 0.75$ & 36.898 & 0.001 \\
varnish & 15 days & 20 & $5.81 \pm 1.70$ & & \\
& 30 days & 20 & $4.06 \pm 0.30$ & & \\
\hline
\end{tabular}

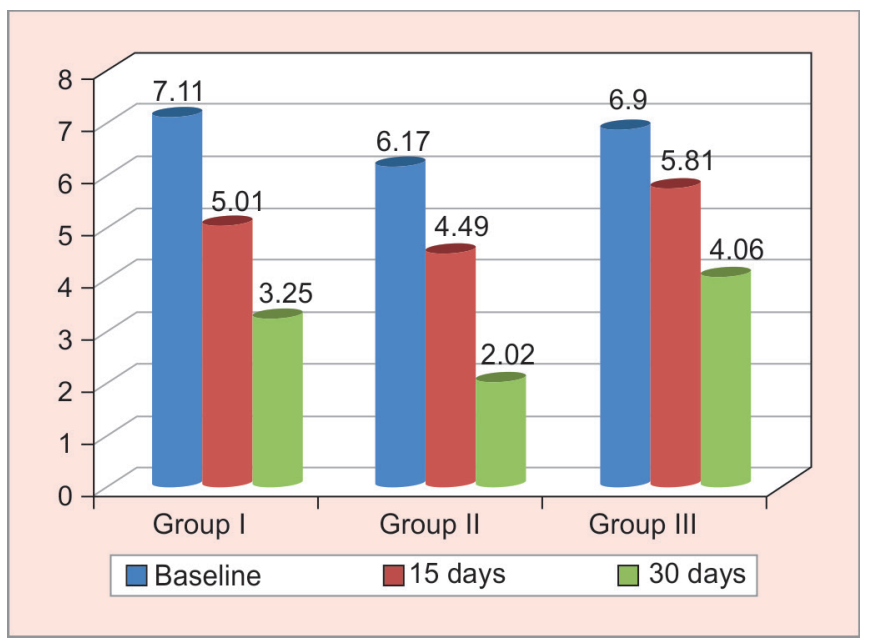

Fig. 4: Mean VAS scores among three groups for cold air blast stimulus 
The occurrence of DH varies hugely (range, 4-74\%), owing to the variations in population and diverse investigation methods. In the present study, most patients suffering from $\mathrm{DH}$ are aged between 36 and 45 years, which are similar to the study conducted by Flynn et al. ${ }^{11}$ in the population of West of Scotland. A higher incidence of $\mathrm{DH}$ has been reported in females than in males in our study, which is in accordance with the trial conducted by Miglani et al., ${ }^{8}$ who also reported a higher incidence of $\mathrm{DH}$ in women patients.

In this study, the fluoride varnish treatment group demonstrated the least reduction of DH. Merika et al. ${ }^{12}$ and Ritter et al. ${ }^{13}$ specified that clinician-applied fluoride varnish treatment has been widely used to lower $\mathrm{DH}$ with an immediate and significant pain relief that lasts for several weeks. Dental varnish has an exceptional property of staying on tooth surfaces for hours, thus necessitating the varnish base to infiltrate deep into dentinal tubules and obstruct the movements of fluid and they discharge increased concentrations of fluoride ions, forming $\mathrm{CaF}_{2}$ over a substantial period of time.

$\mathrm{DH}$ has also been treated with lasers. Recently, a low-level DL has been assessed and found to be efficacious. Even though several lasers have been evaluated, DLs have become more popular as they are easily available and economical. DLs can be used mainly in direct and indirect forms. The direct method involves direct application of lasers alone, and the indirect method involves the use of some chemical agents followed by laser application. ${ }^{14}$

The present study did not demonstrate any significant difference in the mean VAS scores at baseline. This suggests the absence of difference among the three groups with pain scores being almost the same level. In this study, we found a maximum reduction in $\mathrm{DH}$ in group II, followed by groups I and III, respectively. These results are the same as those obtained in the study of Kumar and Mehta, ${ }^{15}$ in which they found that lasers showed an instant reduction in root sensitivity. This advocates the use of lasers as an acceptable and effective treatment modality for the faster reduction of root sensitivity.

Asnaashari et al. ${ }^{16}$ and Aranha et al. ${ }^{17}$ have demonstrated low-power laser treatment as an appropriate therapeutic strategy for treating $\mathrm{DH}$, which enhances bio-modulatory effects, reduces pain, and lowers inflammatory processes. When DLs were used for the treatment of $\mathrm{DH}$, a gradual decrease in tactile stimuli (TS) and air blast stimulation was noticed on days 15 and 30, when compared to that at baseline. This is in accordance with a study of Matsumoto et al., ${ }^{18}$ who observed $85 \%$ improvement in $\mathrm{DH}$. An improvement of $69.2 \%$ was seen in the group treated with lasers when compared with other groups by Kumazaki et al. ${ }^{19}$

The present study demonstrated a better reduction of $\mathrm{DH}$ in the APF gel iontophoresis group when compared with the fluoride varnish group. Several theories have explained the mechanisms of iontophoresis-induced desensitization. One mechanism involves the application of electric current to dentin, which leads to the formation of dead tracts followed by the development of reparative dentin. The second mechanism suggests that the electrical current results in an altered sensory mechanism of pain conduction, thus inducing paraesthesia. The third mechanism indicates a probable microprecipitation of calcium fluoride by iontophoresis, which may block the hydrodynamically mediated pain inducing stimuli. ${ }^{20}$

McBride et al. ${ }^{21}$ demonstrated the fluoride concentration to be two times more in iontophoretically treated teeth than in topically treated teeth and 20 times more in the treated group than that in the control teeth. Paine et al. ${ }^{22}$ explained the formation of calcium fluoride, fluorohydroxyapatite, and phosphate ions in teeth treated with APF gel. The phosphoric acid induces a low $\mathrm{pH}$ and favors the formation of fluorohydroxyapatite, thus preventing loss of phosphate ions from the enamel surface. APF iontophoresis may have better infiltration of fluoride ions into the tubules, thus providing relief over a greater period of time.

The shortcoming of the present study is that pain is a variable perception which is different in every individual. Meticulous observation of the groups over longer periods has resulted in the enhanced ability of the present study to identify the differences between treatment groups and to confirm the stability and sustainability of clinical outcomes. We obtained significant results with the chosen sample size; however, a larger sample size would be desirable.

\section{Conclusion}

The present study concluded that a $980 \mathrm{~nm}$ wavelength DL showed significantly greater reduction of $\mathrm{DH}$ followed by iontophoresis with APF and fluoride varnish.

\section{Clinical Significance}

$\mathrm{DH}$ is one of the chief complaints reported in dental clinics. It may develop naturally when the root uncovers to the oral environment due to gingival recession or it may develop sequentially after scaling and root planning and periodontal surgical procedures. Hence, clinicians should know about the most efficacious available therapeutic option for this condition.

\section{References}

1. Sonawane MR, Shah MU, et al. Comparison of clinical efficacy of three commercially available desensitizing mouthwashes in the treatment of dentinal hypersensitivity. I J Pre Clin Dent Res 2015;2(2):20-25.

2. Dababneh $\mathrm{RH}$, Khouri AT, et al. Dentin hypersensitivity-an enigma? A review of terminology, epidemiology, mechanisms, aetiolog and management. Br Dent J 1999;187:606-611. DOI: 10.1038/ sj.bdj.4800345.

3. Leye Benoist F, Niang SO, et al. Treatment of Dentin Hypersensitivity: A Systematic Review of Randomized Clinical Trials. J Dent Oral Care Med 2016;2(2):204. DOI: 10.15744/2454-3276.2.204.

4. Indurkar MS, Maurya AS. The Clinical Effect of Diode Laser Versuslontophoresis With Acidulated Phosphate Fluoride Gel in the Treatment of Dentin Hypersensitivity. Indian J Appl Res 2015;5(7):33-35. DOI: 10.15373/2249555X.

5. IrudayaNirmala J, Ramakrishnan T, et al. Iontophoresis a boon for treatment of dentinal hypersensitivity: case report. Int J Cur Res Rev 2016;8(23):16-20.

6. Scherman A, Jacobsen PL. Managing dentin hypersensitivity: what treatment to recommend to patients. J Am Dent Assoc 1992;123:5761. DOI: 10.14219/jada.archive.1992.0107.

7. Suri I, Singh $\mathrm{P}$, et al. A comparative evaluation to assess the efficacy of $5 \%$ sodium fluoride varnish and diode laser and their combined application in the treatment of dentin hypersensitivity. J Indian Soc Periodontol 2016;20:307-314. DOI: 10.4103/0972-124X.181243.

8. Miglani S, Aggarwal V, et al. Dentin hypersensitivity: Recent trends in management. J Conserv Dent 2010;13(4):218-224. DOI: 10.4103/09720707.73385 .

9. Tailor A, Shenoy N, et al. To compare and evaluate the efficacy of bifluorid 12, diode laser and their combined effect in treatment of dentinal hypersensitivity - a clinical study. NitteUniv J Health Sci 2014;4:54-58.

10. Suri I, Singh $P$, et al. A comparative evaluation to assess the efficacy of $5 \%$ sodium fluoride varnish and diode laser and their 
combined application in the treatment of dentin hypersensitivity. J Indian Soc Periodontol 2016;20(3):307-314. DOI: 10.4103/0972124X.181243.

11. Flynn J, Galloway R, et al. The incidence of hypersensitive teeth in the West of Scotland. J Dent 1985;13:230-236. DOI: 10.1016/03005712(85)90004-16.

12. Merika K, HeftitArthur F, et al. Comparison of two topical treatments for dentine sensitivity. Eur J Prosthodont Restor Dent 2006;14(1):38-41.

13. Ritter AV, de L Dias W, et al. Treating cervical dentin hypersensitivity with fluoride varnish: a randomized clinical study. J Am Dent Assoc 2006;137(7):1013-1020. DOI: 10.14219/jada.archive.2006.0324.

14. Ferreira AN, Silveira L, et al. Effect of GaAlAs laser on reactionaldentinogenesis induction in human teeth. Photomed Laser Surg 2006;24:358-365. DOI: 10.1089/pho.2006.24.358.

15. Kumar NG, Mehta DS. Short-term assessment of the Nd:YAG laser with and without sodium fluoride varnish in the treatment of dentin hypersensitivity - a clinical and scanning electron microscopy study. J Periodontol 2005;76:1140-1147. DOI: 10.1902/jop.2005.76.7.1140.
16. Asnaashari $M$, Moeini $M$. Effectiveness of lasers in the treatment of dentin hypersensitivity. J Lasers Med Sci 2013;4:1-7.

17. Aranha AC, Eduardo Cde P. Effects of Er: YAG and Er, Cr: YSGG lasers on dentine hypersensitivity. Short-term clinical evaluation. Lasers Med Sci 2012;27:813-818. DOI: 10.1007/s10103-011-0988-9.

18. Matsumoto K, Funai $H$, et al. Study on the treatment of hypersensitive dentine by GaAlAs laser diode. J Conserv Dent 1985;28:766-771.

19. Kumazaki M, Zennyu K, et al. Clinical evaluation of GaAlAssemiconductor laser in the treatment of hypersensitive dentin. J Conserv Dent 1990;33:911-918.

20. Aparna S, Setty $S$, et al. Comparative efficacy of two treatment modalities for dentinal hypersensitivity: a clinical trial. Indian J Dent Res 2010;21:544-548. DOI: 10.4103/0970-9290.74213.

21. McBride MA, Gilpatrick RO, et al. The effectiveness of sodium fluoride iontophoresis in patients with sensitive teeth. Quintessence Int 1991;22:637-640.

22. Paine M, Slots J, et al. Fluoride use in periodontal therapy - Review. J Am Dent Assoc 1998;129:69-77. DOI: 10.14219/jada.archive.1998. 0023. 